\title{
EFEITOS DO ALONGAMENTO PASSIVO NO DESEMPENHO DE SÉRIES MÚLTIPLAS NO TREINAMENTO DE FORÇA
}

\author{
EFFECTS OF PASSIVE STRETCHING PROTOCOL ON MULTIPLE SETS PERFORMANCE \\ IN RESISTANCE TRAINING
}

Artigo Original

Original ARTICLE

Artículo Original

Charles Ricardo Lopes ${ }^{1,3}$

(Educador Físico)

Enrico Gori Soares ${ }^{1}$ (Educador Físico)

André Luis Rodrigues Santos ${ }^{1}$

(Educador Físico)

Marcelo Saldanha Aoki ${ }^{4}$

(Educador Físico)

Paulo Henrique Marchetti ${ }^{1,2}$

(Educador Físico)

1. Universidade Metodista de Piracicaba (UNIMEP) Faculdade de Ciências da Saúde (FACIS), Piracicaba, SP, Brasil.

2. Universidade de São Paulo, Faculdade de Medicina Instituto de Ortopedia e Traumatologia. São Paulo, SP, Brasil.

3. Faculdade Adventista de Hortolândia, Hortolândia, SP, Brasil. 4. Universidade de São Paulo, Escola de Artes, Ciências e Humanidades, São Paulo, SP, Brasil.

\section{Correspondência:}

Paulo H. Marchetti. Programa de

Pós-Graduação em Ciências do

Movimento Humano, Faculdade

de Ciências da Saúde, Universidade

Metodista de Piracicaba.

Rodovia do Açúcar Km 156,

Bloco 7, Sala 42, Taquaral. Piracicaba,

SP, Brasil. 13423-070.

dr.pmarchetti@gmail.com

\section{RESUMO}

Introdução: Recentemente, a prática de exercícios de alongamento tem sido relacionada a déficit no desempenho de atividades força-dependentes. Entretanto, pouco se sabe sobre seu efeito ao longo de séries múltiplas no treinamento de força. Objetivo: Determinar o efeito do alongamento passivo extensivo no desempenho de séries múltiplas e da dor percebida no treinamento de força. Métodos: A amostra foi composta de 12 homens experientes com treinamento de força. O protocolo consistiu de três sessões. A primeira serviu como familiarização e determinação de 10 repetições máximas no supino reto (T1ORM). A segunda e terceira condições foram aleatorizadas entre os sujeitos, sendo uma realizada com a avaliação do supino reto (SR) sem alongamento prévio (SEMA), e a outra com alongamento prévio (COMA). O protocolo de alongamento consistiu de seis séries de 45 seg de alongamento por 15 seg de intervalo visando o músculo peitoral maior. A intensidade do alongamento foi mantida entre 70 e $90 \%$ da percepção subjetiva de desconforto. Antes e após o exercício, a dor local foi avaliada por meio de breve alongamento e palpação. A Análise de Variância ANOVA (2x6) com medidas repetidas foi utilizada para comparar o número de repetições. One-way ANOVA foi utilizada para comparar a carga da primeira série de cada condição (T1ORM/SEMA/COMA). O teste- $t$ de Student foi utilizado para comparar a carga total com a dor. Resultados: Observou-se decréscimo no número de repetições ao longo das séries em ambas as condições, sem diferenças entre as mesmas, para a carga da primeira série, a carga total e a dor. Conclusão: O número de repetições em séries múltiplas é diminuído na mesma magnitude com e sem a realização do alongamento prévio, além de não alterar a carga total e a dor percebida.

Palavras-chave: exercício, treinamento de resistência, desempenho atlético.

\section{ABSTRACT}

Introduction: Stretching exercises have been related to a performance deficit on strength-dependent activities. However, little is known about its effect on multiple sets of strength training. Objective: To determine the effect of an extensive stretching protocol on the performance of multiple sets and soreness in strength training. Methods: The sample was composed by 12 trained-men, experienced in strength training. The protocol consisted of three sessions. The first session was used as a familiarization and load determination to 10 maximum repetitions (T1ORM). The second and third conditions were randomized between subjects, and the bench press exercise (BP) was evaluated without previous stretching protocol (SEMA), and with previous stretching protocol (COMA). The stretching protocol consisted of six sets of $45 \mathrm{sec}$ of stretch with $15 \mathrm{sec}$ of resting intervals to the pectoralis major muscle. The stretching intensity was kept between 70 and $90 \%$ of the subjective point of discomfort. The local soreness was evaluated before and after exercise by a brief stretching and palpation. The Analysis of Variance ANOVA (2x6) was used to compare the maximum number of repetitions. One-way ANOVA was used to compare the load of the first set of each condition (T1ORM/SEMA/COMA). The Student t-test was used to compare the total load and soreness. Results: A decrease on maximum number of repetitions along the sets was observed in both conditions, without differences between them, for the load of the first set, total load and soreness. Conclusion: The number of repetitions in multiple sets was reduced in the same magnitude in the conditions with and without previous stretching; besides, it does not alter the total load or soreness.

Keywords: exercise, resistance training, athletic performance.

\section{RESUMEN}

Introducción: Recientemente, la práctica de ejercicios de alongamiento ha sido relacionada a un déficit en el desempeño de las actividades dependientes de fuerza. Entretanto, poco se sabe sobre su efecto a lo largo de series múltiples en el entrenamiento de fuerza. Objetivo: Determinar el efecto del alongamiento pasivo extensivo en el desempeño de series múltiples y del dolor percibido en el entrenamiento de fuerza. Métodos: La muestra fue compuesta por 12 hombres con experiencia en el entrenamiento de fuerza. El protocolo consistió en tres sesiones. La primera sirvió como familiarización y determinación de 10 repeticiones máximas en el supino recto (T1ORM). La segunday tercera condiciones fueron randomizadas entre los sujetos, siendo una realizada con la evaluación del supino recto 
(SR) sin alongamiento previo (SEMA), y la otra con alongamiento previo (COMA). El protocolo de alongamiento consistió en seis series de 45" de alongamiento por 15" de intervalo con vistas al músculo pectoral mayor. La intensidad del alongamiento fue mantenida entre 70 y $90 \%$ de la percepción subjetiva de incomodidad. Antes y después de las condiciones, fue evaluado el dolor local mediante un breve alongamiento y palpado. Una ANOVA $(2 \times 6)$ con medidas repetidas fue utilizada para comparar el número de repeticiones. Una ANOVA unidireccional fue utilizada para comparar la carga de la primera serie de cada condición (T1ORM/SEMA/COMA). Fue usado el Test t de Student para comparar la carga total y el dolor. Resultados: Se observó disminución del número de repeticiones a lo largo de la serie en ambas condiciones, sin diferencias entre las mismas, para la carga de la primera serie, la carga total y el dolor. Conclusión: El número de repeticiones en series múltiples es disminuido en la misma magnitud con y sin la realización de alongamiento previo, además de no alterar la carga total y el dolor percibido.

Palabras clave: ejercicio, entrenamiento de fuerza, desempeño.

\section{INTRODUÇÃO}

É prática comum de atletas e praticantes recreacionais de atividades físicas e esportivas a incorporação de exercícios de alongamento e de força a fim de melhorar a amplitude de movimento (ADM), a força e hipertrofia muscular ${ }^{1,2}$. Tipicamente em sessões de treinamento de força, um elevado volume (produto das séries e repetições) e carga de treino (produto das séries, repetições e sobrecarga levantada), associado à uma alta intensidade, são necessários a fim de exaurir a musculatura e consequentemente promover os ajustes agudos e adaptações crônicas necessárias ${ }^{3,4}$. Normalmente, em sessões de treinamento divididas por grupo muscular, são utilizados de dois a cinco exercícios para cada grupamento muscular, sendo que para indivíduos treinados pode atingir até 20-25 séries para cada grupo muscular em uma única sessão.

A prática do alongamento passivo como parte da preparação ou sessão de treino tem sido relacionada a um déficit no desempenho de atividades força-dependentes ${ }^{1}$. A literatura científica aponta certos "possíveis mecanismos" responsáveis pela redução na força avaliada como (i) a inibição dos estímulos excitatórios de motoneurônios alfa, via estimulação das terminações nervosas III, IV e órgão tendinoso de Golgi, (ii) aumento do atraso eletromecânico devido a maior complacência da unidade músculo-tendínea, (iii) alteração na relação comprimento-tensão da unidade músculo-tendínea, e (iv) isquemia local devido ao menor fluxo sanguíneo e acúmulo de metabólitos ${ }^{1,2,5}$. Coletivamente, tais efeitos podem levar a diminuição do desempenho em atividades onde um alto recrutamento de unidades motoras é necessário como testes de força máxima ${ }^{6}$ ou exercícios dependentes da utilização dos mecanismos associados ao ciclo alongamento-encurtamento como testes dinâmicos máximos, saltos e arremessos ${ }^{2,7}$.

Poucos foram os estudos encontrados na literatura científica que investigaram os efeitos agudos do alongamento passivo extensivo no desempenho de séries múltiplas e na dor percebida. Nelson et al. ${ }^{8}$, investigaram o efeito de uma sessão de alongamento estático (dois exercícios realizados com quatro séries de 30 segundos para os músculos isquiotibiais) no desempenho de repetições máximas no exercício cadeira flexora e cargas ajustadas entre 40 e $60 \%$ do peso corporal. Seus resultados revelaram um decréscimo no número de repetições máximas realizadas quando o alongamento passivo estático foi empregado. Entretanto, seu estudo investigou apenas o efeito do alongamento em uma única série, sendo que como descrito previamente, sugere-se o emprego de séries múltiplas para a melhora da resistência de força, potência e força máxima9 ${ }^{9}{ }^{10}$. Posteriormente, Ribeiro et al. ${ }^{11}$, não observou diferenças no número de repetições máximas realizadas entre dois protocolos (com e sem a realização de alongamento prévio) em quatro séries realizadas até a falha concêntrica no exercício supino reto, a $80 \%$ de $1 \mathrm{RM}$ e intervalo de dois minutos entre séries. O referido estudo utilizou protocolo de alongamento passivo com uma série de 30 segundos para os grupos musculares alvo. O protocolo foi realizado de maneira unilateral, iniciando pelo grupamento muscular do peitoral maior em seguida realizou-se o alongamento do tríceps braquial.

Devido aos efeitos neuromecânicos tempo-dependentes do tecido biológico ${ }^{12}$ têm-se sugerido que durações totais superiores há 90 segundos devem ser empregadas para que ocorram alterações no desempenho possam ser observadas. Portanto, o objetivo do presente estudo foi determinar o efeito do alongamento passivo extensivo no desempenho de séries múltiplas e da dor percebida no treinamento de força.

\section{MÉTODOS}

O tamanho da amostra foi determinado a partir de um estudo piloto previamente realizado, utilizando indivíduos que possuíam as mesmas características daquelas que foram empregadas no presente estudo, baseando-se em uma significância de 5\% e um poder do teste de $80 \%$. A amostra foi composta por 12 homens saudáveis (idade: $24 \pm 4$ anos, estatura: $176 \pm 5 \mathrm{~cm}$, massa: $82 \pm 11$ kg, distância biacrômial: $37 \pm 1$ cm, 10RM no supino: $679 \pm 177$ N). Todos os sujeitos foram treinados em musculação a pelo menos um ano, e com experiência na realização do exercício supino reto, e sem qualquer acometimento ósteomioarticular (lesão/cirurgia) prévio no membro superior e/ou tronco. Todos os sujeitos foram informados dos procedimentos experimentais, leram e assinaram o Termo de Consentimento Livre e Esclarecido aprovado pelo Comitê de Ética em Pesquisa da Universidade (Protocolo \#39/13).

\section{Design Experimental}

Os voluntários apresentaram-se no laboratório em três sessões, espaçadas de no mínimo 72 horas. Na primeira sessão foram obtidos dados antropométricos (massa, estatura e distância biacrômial) e dados pessoais (idade e tempo de treinamento) por meio de um questionamento oral. Logo após a avaliação inicial, os sujeitos foram familiarizados com os procedimentos experimentais e com o exercício utilizado. Então, um teste para 10 repetições máximas (10RM) foi realizado visando definir a sobrecarga no exercício supino reto na barra guiada.

A segunda e terceira sessões foram aleatorizadas entre os sujeitos, sendo que ambas consistiram de seis séries até a falha muscular concêntrica utilizando 10RM no exercício supino reto na barra guiada. Ambas as sessões foram distinguidas pela inclusão (COMA), ou não (SEMA) de um protocolo de alongamento extensivo antes das séries múltiplas do exercício supino reto. Todas as séries foram realizadas em cadência definida por metrônomo a 40bpm, e um intervalo de um minuto de descanso foi fornecido entre séries. Antes e após a realização de cada 
condição experimental, a dor proveniente do músculo peitoral maior foi avaliada por meio do alongamento e palpação, realizada sempre pelo mesmo pesquisador.

Teste de Repetições Máximas (10RM): O teste de repetições máximas $^{13}$ para 10RM foi realizado para o exercício supino e serviu como controle para as demais condições. Cada sujeito teve no máximo cinco tentativas para cada exercício em cadência previamente selecionada a $40 \mathrm{bpm}$. Foi adotado um intervalo de cinco minutos entre cada tentativa e 30 minutos entre cada exercício. Para o supino reto foi adotada uma empunhadura de $200 \%$ da distância biacromial ${ }^{5,6}$. Os voluntários partiram com os cotovelos estendidos, desceram a barra controladamente até atingir aproximadamente $90^{\circ}$ de flexão de cotovelos, e assim retornaram a posição inicial com os cotovelos estendidos. Não foram permitidas paradas na execução do movimento entre as fases ascendentes e descendentes e somente foram válidas as séries em que a correta execução dos exercícios foi realizada. Para minimizar erros nos testes foram adotadas as seguintes estratégias: (i) os sujeitos receberam informações sobre a técnica adequada de cada exercício antes dos testes (ii) a execução da técnica do exercício foi monitorada e corrigida quando necessário (iii) os sujeitos foram sistematicamente encorajados verbalmente durante os testes.

Análise da dor percebida (DOR): Os sujeitos permaneceram em pé com uma das mãos alinhadas ao ombro e apoiadas em um objeto fixo. Passivamente, um dos pesquisadores alongou brevemente o grupo muscular do peitoral maior realizando uma abdução horizontal de ombro, em seguida apalpou o músculo peitoral maior do sujeito. Os sujeitos foram orientados a reportar o nível de dor localizada no músculo frente à palpação do pesquisador antes do protocolo de alongamento (quando necessário) e após as seis séries de realização do supino reto. Foi utilizada uma escala de 0 a 10 unidades arbitrárias (UA). Onde $0=$ "sem dor" e 10="máximo de dor imaginável após a realização de um treino de musculação"14.

Protocolo de alongamento estático extensivo (AEE): Os sujeitos permaneceram sentados em uma cadeira, com as mãos atrás da cabeça, braços acima da articulação do ombro. Então, um pesquisador moveu ambos os braços (complexo articular do ombro), passivamente, para a máxima ADM em abdução horizontal, trazendo a articulação do cotovelo para trás o máximo possível. O protocolo de AEE empregado consistiu de seis séries de 45 segundos de duração com 15 segundos de intervalo entre cada alongamento ${ }^{1,2}$. A intensidade foi de $70-90 \%$ da percepção subjetiva de desconforto (PSD), onde $0=$ "sem desconforto de alongamento" e 100\%="o máximo desconforto de alongamento imaginável"1,2,15. A intensidade do PSD foi perguntada para todos os sujeitos durante o protocolo de AEE. Durante todos os alongamentos, a ADM foi reajustada a 70-90\% do PSD se necessário. O protocolo de alongamento foi aplicado e controlado pelo mesmo pesquisador.

\section{Análise dos dados}

A carga total foi calculada a partir do produto do número de séries, repetições, sobrecarga utilizada no teste de 10RM. Cinco análises de desempenho foram realizadas: (i) comparar as condições SEMA e COMA no número de repetições máximas em cada uma das seis séries do supino reto, (ii) comparar o índice de fadiga entre a primeira e última série de cada uma das condições pela fórmula proposta por Ribeiro et al.:11,

$$
I F=\frac{N^{\circ} \operatorname{Rep}\left(1^{\mathrm{a}} \text { série }\right)-N^{\circ} \operatorname{Rep}\left(6^{\mathrm{a}} \text { série }\right)}{N^{\circ} \operatorname{Rep}\left(1^{\mathrm{a}} \text { série }\right)} \times 100
$$

onde IF significa índice de fadiga e NRep significa número de repetições máximas realizadas; (iii) comparar a carga da primeira série de cada um dos protocolos (SEMA e COMA) com o valor do teste de 10RM (T10RM) a fim de verificar o efeito imediato do alongamento; (iv) comparar a carga total das condições SEMA e COMA, sendo calculado pela somatória individual de cada série; e (v) comparar o número total de repetições realizadas das condições SEMA e COMA calculado pela somatória total do número de repetições de cada série. Os dados da dor percebida foram tabulados e comparados nas duas condições.

\section{Análise Estatística}

Uma análise descritiva com valores de média e desvio padrão foi utilizada para análise dos dados. A normalidade e homogeneidade das variâncias foram verificadas utilizando o teste de Shapiro-Wilk e de Levene, respectivamente. Foi utilizado um teste $t$ de student pareado para amostras dependentes na comparação do load total, número de repetições total e índice de fadiga entre as condições SEMA e COMA. Uma ANOVA $(2 \times 6)$ de medidas repetidas, com os fatores (condição $x$ série), foi utilizada para comparar as diferenças nas repetições máximas de cada condição. Uma ANOVA (2×2) de medidas repetidas, com os fatores (condição $x$ tempo), foi utilizada para comparar diferenças na dor percebida. Uma one-way ANOVA foi utilizada para comparar a carga da primeira série nas condições (T10RM, SEMA e COMA). Um post hoc de Bonferroni (com correção) foi utilizado para verificar as diferenças quando necessário. O cálculo do tamanho do efeito (TE) foi realizado através da fórmula de Cohen e os resultados basearam-se nos seguintes critérios: $<0,35$ efeito trivial; 0,35-0,80 pequeno efeito; 0,80-1,50 efeito moderado; e >1,50 grande efeito, para sujeitos treinados recreacionalmente baseado em Rhea ${ }^{16}$. Significância (a) de 5\% foi utilizada para todos os testes estatísticos, através do software SPSS versão 21.0.

\section{RESULTADOS}

Desempenho de repetições máximas no protocolo de séries múltiplas: Foi verificada uma redução significante no número máximo de repetições entre a primeira série e as demais nas condições SEMA e COMA $(P<0,01)$. Entretanto, comparando-se a primeira série entre condições não foram observadas diferenças significantes $(P>0,05 ; \mathrm{TE}=0.73$ [efeito pequeno]; $\Delta \%=15,9 \%)$. O número de repetições da segunda série na condição SEMA foi significantemente maior que séries seguintes na mesma condição $(P<0,05)$. Na condição COMA a segunda série foi significantemente maior somente da sexta série na mesma condição $(P<0,01)$. Entretanto, similarmente à primeira série não foram observadas diferenças entre condições ( $P=0,36 ; \mathrm{TE}=1,16$ [efeito moderado]; $\Delta \%=29,1 \%)$. Somente a terceira série da condição SEMA foi significantemente maior que a sexta série na mesma condição $(P<0,05)$. Não foram observadas diferenças significantes entre condições na terceira $(P<0,05 ;$ TE $=0,25$ [efeito trivial]; $\Delta \%=10,6 \%)$, quarta $(P=1,0 ;$ TE $=0,07$ [efeito trivial]; $\Delta \%=3,5 \%)$, quinta $(P=1,0 ; \mathrm{TE}=0,06$ [efeito trivial]; $\Delta \%$ $=3,5 \%)$ e sexta série $(P<0,05 ; \mathrm{TE}=0,06$ [efeito trivial]; $\Delta \%=4,0 \%)$. As diferenças no desempenho são apresentadas na figura 1.

Índice de fadiga: O cálculo do índice de fadiga não diferiu entre condições (SEMA: 80,21 \pm 15,81\%, COMA: 73,05 \pm 16,87\%; $P=0,23$; $\mathrm{TE}=0,43$ [efeito pequeno]; $\Delta \%=8,9 \%$ ).

Comparação da carga da primeira séries das condições COMA e SEMA e T10RM: Não foram observadas diferenças significantes na primeira série entre as condições (T10RM: $6794 \pm 1773$ N; SEMA: $6791 \pm 1533$ N; COMA: $5715 \pm 2156, P>0,05)$. O cálculo do tamanho do efeito revelou um efeito trivial ( $T E=0,001 ; \Delta \%=0,05 \%$ ) entre as condições T10RM e SEMA. Um pequeno efeito foi observado na comparação entre as condições T10RM e COMA (TE=0,54; $\triangle \%=15,8 \%)$ e SEMA e COMA ( $\mathrm{TE}=0,57 ; \Delta \%=15,8 \%$ ). 
Carga total: A carga total não diferiu significantemente entre condições (SEMA: $17406 \pm 3311 \mathrm{~N}$ e COMA $15191 \pm 3322 \mathrm{~N}, P=0,12 ; \mathrm{TE}=0,66$ [efeito pequeno]; $\Delta \%=12,7 \%$ ).

Número total de repetições (RM): Foi verificada diferença significante no número total de repetições (somatória do número de repetições realizadas nas seis séries) entre as condições (SEMA: $25 \pm 3$ RM e COMA: $22 \pm 3 \mathrm{RM} ; P=0,01 ; \mathrm{TE}=1,0$ [efeito moderado]; $\triangle \%=12,0 \%$ ), (figura 2).

Dor percebida: Não foram observadas diferenças entre as condições em nenhum dos momentos analisados $(P>0,05)$ (tabela 1).

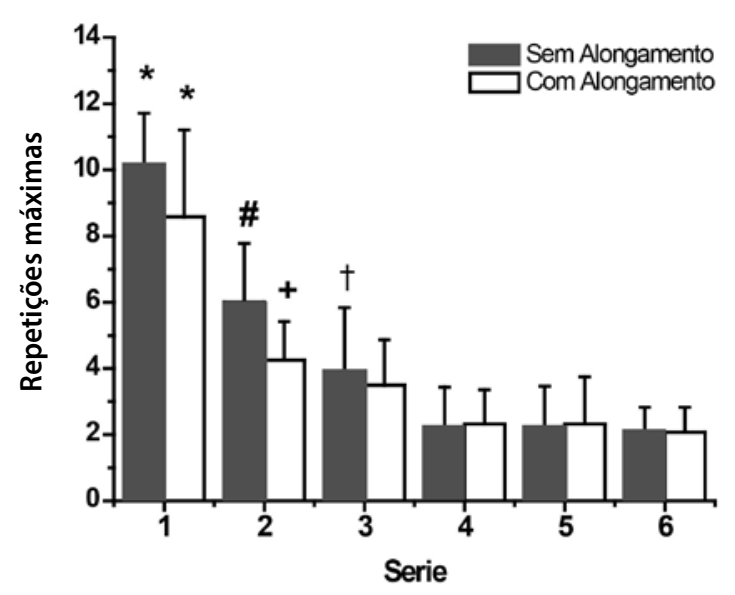

* diferença significativa entre a 1a série e as demais na mesma condição $(P<0,01)$; \# diferença significativa da 2a série para a 3a, 4a, 5a e 6a série na mesma condiçăo $(P<0,05) ;+$ diferença significativa entre a $2 a$ e $6 a$ série na mesma condiçăo $(P<0,01) ; \dagger$ diferença significativa entre $3 a$ e $6 a$ série na mesma condição $(P<0,05)$

Figura 1. Média e desvio padrão do número máximo de repetições realizadas nas condições com alongamento (COMA) e sem alongamento (SEMA), $n=12$.

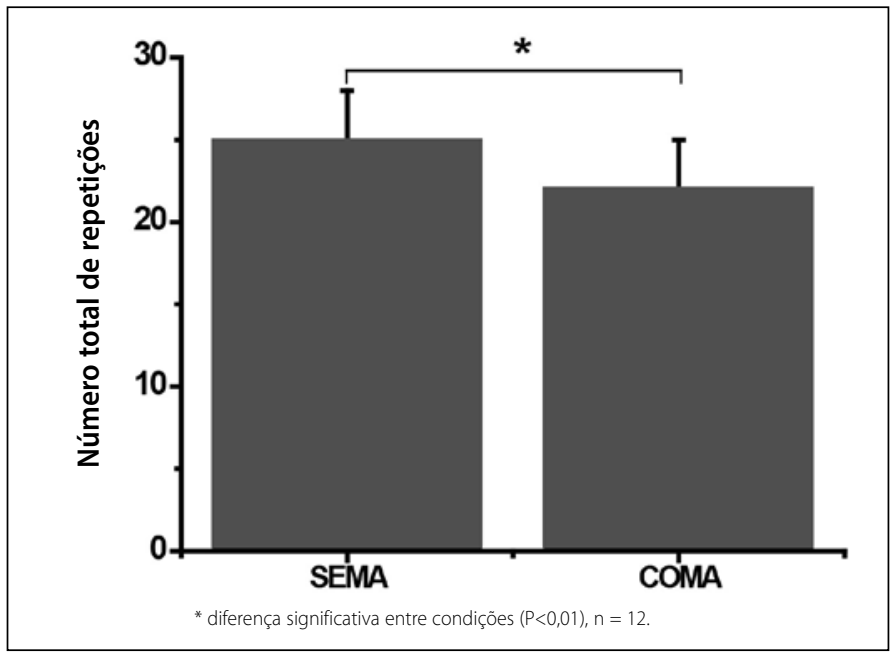

Figura 2. Média e desvio padrão do número total de repetições realizadas nas condições com alongamento (COMA) e sem alongamento (SEMA).

Tabela 1. Comparação da dor percebida entre momentos e condições avaliadas, $n=12$.

\begin{tabular}{c|c|c|c}
\hline $\begin{array}{c}\text { Comparação } \\
\text { (Média } \pm \text { DP) UA }\end{array}$ & Valor de $\boldsymbol{P}$ & $\begin{array}{c}\text { Tamanho } \\
\text { do efeito }\end{array}$ & $\Delta \%$ \\
\hline $\begin{array}{c}\text { Pré-SEMA } \times \text { Pré-COMA } \\
(2,3 \pm 2,9) \times(1,0 \pm 2,1)\end{array}$ & 0,5 & 0,51 (pequeno) & $56 \%$ \\
\hline $\begin{array}{c}\text { Pré-SEMA } \times \text { Pós-SEMA } \\
(2,3 \pm 2,9) \times(2,2 \pm 3,3)\end{array}$ & $>0,05$ & 0,03 (trivial) & $4 \%$ \\
\hline $\begin{array}{c}\text { Pré-COMA } \times \text { Pós-COMA } \\
(1,0 \pm 2,1) \times(1,4 \pm 2,5)\end{array}$ & $>0,05$ & 0,17 (trivial) & $28 \%$ \\
\hline $\begin{array}{c}\text { Pós-SEMA } \times \text { Pós-COMA } \\
(2,2 \pm 3,3) \times(1,4 \pm 2,5)\end{array}$ & $>0,05$ & 0,27 (trivial) & $36 \%$ \\
\hline
\end{tabular}

Legenda: UA - unidade arbitrária.

\section{DISCUSSÃO}

Inicialmente, observou-se uma queda no número total de repetições (somatória do número de repetições máximas realizadas nas seis séries) para a condição COMA em comparação com SEMA. Adicionalmente, apesar de não ter sido observada diferença estatística, verificou-se queda de 12,7\% na carga total para a condição COMA, apresentando pequeno tamanho de efeito em comparação com a condição SEMA. Diversas evidências científicas sugerem uma redução no desempenho de atividades força-dependentes quando precedidas por protocolos de alongamento estático, principalmente aqueles que apresentam um volume total acima de 90 segundos, e intensidades além do ponto de desconforto 1,17,18. Tal diminuição do desempenho tem sido demonstrada em testes de força máxima isométrica ${ }^{6,19}$, e exercícios dependentes da utilização dos mecanismos associados ao ciclo alongamento-encurtamento como testes dinâmicos máximos, saltos e arremessos ${ }^{2,7}$. Quanto aos membros superiores, Leone et $a l^{19}{ }^{19}$, investigaram homens fisicamente ativos e observaram uma redução na força voluntária máxima isométrica, associada à uma diminuição da atividade muscular do peitoral maior (porção esternocostal) e tríceps braquial (cabeça longa e lateral) após a realização de dois exercícios específicos para os adutores horizontais do ombro e extensores do cotovelo com um protocolo de alongamento estático de duas séries de 30 segundos de. Nelson et al. ${ }^{8}$, investigaram homens e mulheres fisicamente ativos realizaram um estudo onde um protocolo de alongamento composto de dois exercícios para os flexores do joelho com quatro séries de 30 segundos de alongamento e 15 segundos de intervalo para cada exercício, reportaram um decréscimo de $24 \%$ no número de repetições máximas realizadas no exercício mesa flexora utilizando uma sobrecarga de $60 \%$ do peso corporal. Adicionalmente, quando o exercício foi realizado com $40 \%$ do peso corporal foi observada redução de $9 \%$ no número de repetições máximas em comparação com sua realização sem o alongamento prévio. Paulo et al. ${ }^{20}$, reportaram decréscimo na força máxima (Teste de carga máxima [1RM]) nos exercícios supino reto e agachamento e redução no número de repetições máximas utilizando $70 \%$ de 1 RM no exercício supino reto quando os mesmos foram precedidos de um protocolo composto de seis exercícios de alongamento passivo utilizando três séries de 30 segundos com para os principais grupos musculares envolvidos em cada tarefa, totalizando 18 minutos de alongamento para cada segmento corporal. Entretanto, os mesmos diferem do presente estudo devido ao fato de utilizarem somente uma tarefa máxima ou tarefas submáximas (\%1RM) na avaliação do efeito agudo do alongamento. Poucos estudos foram encontrados na literatura científica que investigam o efeito do alongamento estático em atividades submáximas realizadas até a falha muscular concêntrica como no caso do presente estudo (ex. 10RM).

As alterações mecânicas e neurofisiológicas decorrentes da prática de alongamento têm sido reportadas como os principais mecanismos responsáveis pela queda de desempenho nas atividades força-dependentes. Dentre as alterações mecânicas, a redução da rigidez da unidade músculo tendínea (UMT) causada pelo alongamento 1,21 pode ter reduzido a transferência de energia entre as fases excêntricas e concêntricas do exercício, bem como ter alterado as relações de comprimento-tensão da UMT afetadas pelo alongamento estático. Wilson et al. ${ }^{22}$, reportaram uma correlação positiva entre a força máxima concêntrica do supino reto e a rigidez da UMT. Portanto, é possível que o protocolo de alongamento utilizado tenha diminuído o desempenho no exercício supino reto devido à correlação entre a produção de força e a rigidez da UMT. Paralelamente aos efeitos mecânicos, uma inibição da atividade muscular tem sido observada após a realização de protocolos de alongamento estático 1,2,19. Sendo que os principais fatores 
responsáveis pela queda da atividade muscular estão relacionados à inibição local dos motoneurônios alfa podendo ou não ser influenciada pelos órgãos tendinosos de Golgi, e/ou associada a uma queda na excitabilidade dos neurônios aferentes la, causada pelos receptores tipos III e IV². A combinação de tais mecanismos pode afetar negativamente a produção de força final durante a execução do exercício supino reto.

O número de repetições máximas realizadas em cada uma das séries não diferiu entre condições, entretanto, foi observada queda no número de repetições na condição COMA de 15,97\% (TE=pequeno) e 29,1\% (TE=moderado) na primeira e segunda série respectivamente em comparação com a condição SEMA. Somente efeitos triviais na comparação entre condições foram observadas nas séries seguintes. Similarmente ao número de repetições, não foi observada diferença significante na carga da primeira série entre condições (SEMA, COMA e T10RM). Entretanto, foi observado um pequeno tamanho de efeito e uma redução de 15,8\% da carga na condição COMA em comparação com as demais condições. Coletivamente, tais resultados sugerem que os maiores efeitos deletérios do alongamento foram observados nas séries iniciais do protocolo, mas podem ser diluídos ao longo do tempo ${ }^{21,23,24}$. Apesar do volume total do alongamento diferir da prática esportiva, Fowles et al. ${ }^{24}$, reportaram um decréscimo na contração voluntária máxima (CVM) dos flexores plantares de até uma hora (9\% de decréscimo) após a realização em 13 séries de 135 segundos (totalizando 30 minutos em alongamento). Entretanto, entre 80\%-87\% da contração voluntária máxima foi restaurada de 5 a 10 minutos após a realização do alongamento estático. Adicionalmente, a atividade muscular retornou aos valores basais 15 minutos após, enquanto que a relação torque-ângulo foi alterada somente logo após a realização do protocolo de alongamento. Bradley et al. ${ }^{23}$, investigaram o time-course do alongamento estático na altura do salto vertical, reportou queda no desempenho somente após a realização de um protocolo de alongamento estático. Cinco minutos após a realização do alongamento estático os valores basais foram reestabelecidos. Seu protocolo de alongamento consistiu em quatro séries de 30 segundos onde cinco exercícios para os principais grupos musculares envolvidos no salto vertical. Adicionalmente aos estudos demonstrados, Taniguchi et al. ${ }^{21}$, observaram que a rigidez apresentada pelo músculo gastrocnêmio lateral e medial foi diminuída, e a amplitude articular em dorsiflexão de tornozelo foi aumentada após cinco séries de um minuto de alongamento passivo, porém tais medidas gradualmente retornam aos valores basais em até 20 minutos após o protocolo de alongamento. Portanto, de acordo com os resultados encontrados no presente estudo e nos estudos de Fowles et al. ${ }^{24}$, Bradley et al. ${ }^{23}$, e Taniguchi et al..21 , os maiores decréscimos no desempenho são observados imediatamente após a realização do alongamento estático, sendo que o retorno da atividade muscular, associada ao aumento gradual da rigidez da UMT podem ter contribuído para a similaridade entre resultados observados nas séries finais de séries múltiplas no exercício supino reto.

Ribeiro et al. ${ }^{11}$, não observaram diferenças no número de repetições até a falha concêntrica durante a realização de quatro séries de supino reto (com 80\% de 1RM) em condições com e sem alongamento estático dos adutores horizontais de ombro e extensores de cotovelo. Foi observado um decréscimo no número de RMs ao longo das séries executadas em ambas as condições. Adicionalmente, não foram observadas diferenças significantes no índice de fadiga calculado entre a primeira e última série. Aparentemente, a prática prévia do alongamento parece não alterar o processo contínuo de instauração da fadiga, como mostram os estudos de Ribeiro et al. ${ }^{11}$, e o presente estudo, além disto não foram observadas alterações no índice de fadiga calculado entre a primeira e última série. O proto- colo de alongamento consistiu na realização de somente uma série de 30 segundos de duração no ponto de desconforto para cada um dos grupos musculares envolvidos. Já o estudo de Martins et al. ${ }^{25}$, investigou o efeito do volume de alongamento estático no número de repetições em três séries de 10RM nos exercícios supino reto e mesa flexora. Quatro condições foram testadas: sem alongamento, 30 segundos de alongamento, 2x30 segundos de alongamento e $3 \times 30$ segundos de alongamento. Os grupos musculares alongados foram os adutores horizontais do ombro e flexores do joelho previamente aos respectivos exercícios. Diferentemente do presente estudo, foi observada redução no número total de repetições em todas as condições onde o alongamento prévio foi realizado. O decréscimo no número total de RM foi relacionado ao volume de alongamento realizado, em que maiores volumes causaram maiores decréscimos no desempenho.

Apesar de reduções percentuais no desempenho terem sido observados no presente estudo, às mesmas não atingiram significância estatística nas comparações entre condições para as variáveis do desempenho de repetições máximas no protocolo de séries múltiplas, índice de fadiga, comparação da carga da primeira série das condições COMA, SEMA e T10RM, e carga total. Possivelmente, a estratégia para o recrutamento das unidades motoras pode ter sofrido alteração a fim de manter o desempenho na execução do supino reto. Estudos, investigando fadiga induzida por exercícios isolados têm demonstrado alterar coordenação intermuscular em exercícios multiarticulares subsequentes ${ }^{26,27}$. Gentil et al. ${ }^{27}$ e Brennecke et al. ${ }^{26}$ demonstraram maior solicitação do tríceps braquial na realização do supino reto após a pré-exaustão prévia dos adutores horizontais do ombro. O presente estudo utilizou o alongamento específico para os adutores horizontais do ombro (peitoral maior e deltóide anterior), possivelmente causando alterações no feedback proprioceptivo (inibição dos motoneurônios alfa) devido à alterações na relação comprimento-tensão da musculatura, diminuindo sua contribuição durante um exercício multiarticular 1,12,15,17. Os estudos previamente apresentados utilizaram alongamentos para todos os grupos musculares envolvidos nos exercícios investigados, impossibilitando solicitação de outros grupamentos musculares na realização da tarefa imposta ${ }^{8,20}$. Portanto, a queda na produção de força causada pelo alongamento específico dos adutores horizontais de ombro possa ter alterado o padrão de recrutamento dos músculos sinérgicos do supino reto, aumentando a solicitação dos extensores do cotovelo a fim de compensar o déficit de força dos adutores horizontais do ombro causada pelo alongamento.

Finalmente, apesar de a prática empírica apoiar o uso do alongamento previamente as atividades esportivas e recreativas a fim de evitar sintomas de dor muscular ou efeitos no desempenho, a literatura científica não apresenta evidências para tal ${ }^{28}$. De fato, existem relatos na literatura científica indicando que somente a realização de alongamento seria capaz de promover dor muscular ${ }^{29}$. Seis mecanismos são propostas a fim explicar as principais causas da dor muscular após a realização de exercícios de força, sendo sua magnitude afetada pela carga de treino utilizada. São eles: acúmulo de íons $\mathrm{H}^{+}$, espasmos musculares, dano no tecido conjuntivo, dano no tecido muscular, inflamação e efluxo de enzimas do retículo sarcoplasmático ${ }^{29}$. Quanto ao presente estudo, não foram observadas diferenças na dor muscular entre condições. Possivelmente, pelo fato de não terem sido observadas diferenças significantes na carga total realizada entre condições, a magnitude de dano tecidual possa ter sido similar. Adicionalmente, foi demonstrado que indivíduos treinados apresentam adaptações neuromusculares específicas a fim de diminuir a magnitude do trauma tecidual em sessões repetidas de treinamento de força, tal mecanismo é conhecido como efeito da carga repetida ${ }^{30}$. 
O presente estudo possui algumas limitações. Possivelmente, o método de análise escolhido (repetições até a falha muscular concêntrica) possa não ter sido sensível o suficiente para detectar pequenos efeitos agudos decorrentes do protocolo de alongamento estático extensivo. Adicionalmente, a falta de verificação dos padrões de atividade muscular, avaliação do ganho de amplitude de movimento do complexo articular do ombro e medias diretas de hipóxia e dano tecidual poderiam ter dado maior embasamento aos resultados obtidos no presente estudo. Portanto, os autores sugerem que pesquisas futuras devam ser realizadas buscando utilizar diferentes equipamentos e métodos de análise na investigação dos diferentes efeitos agudos do alongamento passivo extensivo no desempenho. Adicionalmente, diferentes combinações de métodos (alongamento estático/dinâmico, ativo/passivo, facilitação neuromuscular proprio- ceptiva) e variáveis de carga (volume, intensidade, intervalo) podem ser utilizados a fim de investigar seus efeitos no desempenho de exercícios realizados em séries múltiplas.

\section{CONCLUSÃO}

Conclui-se que o alongamento passivo extensivo não afetou o desempenho de repetições e carga em séries múltiplas na realização do exercício supino reto. Portanto, os dados do presente estudo não suportam a hipótese de que o alongamento extensivo altere o desempenho e a dor muscular percebida em séries múltiplas no treinamento de força.

Todos os autores declararam não haver qualquer potencial conflito de interesses referente a este artigo.

\section{REFERÊNCIAS}

1. Behm DG, Chaouachi A. A review of the acute effects of static and dynamic stretching on performance. Eur J Appl Physiol. 2011;111(11):2633-51.

2. Marchetti PH, Silva FH, Soares EG, Serpa EP, Nardi PS, Vilela Gde B, et al. Upper limb static-stretching protocol decreases maximal concentric jump performance. J Sports Sci Med. 2014;13(4):945-50.

3. Lin J, Chen T. Diversity of strength training methods: A theoretical approach. J Strength Cond Res. 2012;34(2):42-9.

4. Zatsiorsky VM, Kraemer WJ. Ciência e prática do treinamento de força. 2a. ed. São Paulo: Phorte Editora; 2008

5. Marchetti PH, Arruda CC, Segamarchi LF, Soares EG, Ito DT, Luz Junior DA, et al. Exercício supino: uma breve revisão sobre os aspectos biomecânicos. Braz J Sports Exerc Res. 2010;1(2):135-42.

6. Duffey MJ. A biomechanical analysis of the bench press [tesis]. Penssylvania, USA: The Pennsylvania State University; 2008

7. Torres EM, Kraemer WJ, Vingren JL, Volek JS, Hatfield DL, Spiering BA, Ho JY, et al. Effects of stretching on upper-body muscular performance. J Strength Cond Res. 2008;22(4):1279-85.

8. Nelson AG, Kokkonen J, Arnall DA. Acute muscle stretching inhibits muscle strength endurance performance. J Strength Cond Res. 2005;19(2):338-43.

9. Kraemer WJ, Adams K, Cafarelli E, Dudley GA, Dooly C, Feigenbaum MS, et al. American College of Sports Medicine position stand. Progression models in resistance training forhealthy adults. Med Sci Sports Exerc. 2002;34(2):364-80.

10. Kraemer WJ, Ratamess NA. Fundamentals of resistance training: progression and exercise prescription. Med Sci Sports Exerc. 2004;36(4):674-88.

11. Ribeiro AS, Romanzini M, Dias DF, Ohara D, da Silva DR, Achour A Jr, et al. Static stretching and performance in multiple sets in the bench press exercise. J Strength Cond Res. 2014;28(4):1158-63.

12. Serpa EP, Vilela GB, Marchetti PH. Aspectos biomecânicos da unidade músculo-tendínea sob efeito do alongamento. Revista CPAQV. 2014;6(1).

13. Chandler TJ, Brown LE. Conditioning for strength and human performance. 2nd. ed. Vancouver, CB: Wolters Kluwer, 2013.

14. Uchida MC, Nosaka K, Ugrinowitsch C, Yamashita A, Martins E Jr, Moriscot AS, et al. Effect of bench press exercise intensity on muscle soreness and inflammatory mediators. J Sports Sci. 2009;27(5):499-507.

15. Behm DG, Kibele A. Effects of differing intensities of static stretching on jump performance. Eur J Appl Physiol. 2007;101(5):587-94

16. Rhea MR. Determining the magnitude of treatment effects in strength training research through the use of the effect size. J Strength Cond Res. 2004;18(4):918-20.
17. Kay AD, Blazevich AJ. Effect of acute static stretch on maximal muscle performance: a systematic review. Med Sci Sports Exerc. 2012;44(1):154-64

18. Molacek ZD, Conley DS, Evetovich TK, Hinnerichs KR. Effects of low- and high-volume stretching on bench press performance in collegiate football players. J Strength Cond Res. 2010;24(3):711-6.

19. Leone DC, Pezarat P, Valamatos MJ, Fernandes O, Freitas S, Moraes AC. Upper body force production after a low-volume static and dynamic stretching. Eur J Sport Sci. 2014;14(1):69-75.

20. Paulo AC, Ugrinowitsch C, Leite GS, Arsa G, Marchetti PH, Tricoli V. Efeito agudo dos exercícios de flexibilidade no desempenho de força máxima e resistência de força de membros inferiores e superiores. Motriz. 2012;15(2):345-55

21. Taniguchi K, Shinohara M, Nozaki S, Katayose M. Acute decrease in the stiffness of resting muscle belly due to static stretching. Scand J Med Sci Sports. 2015;25(1):32-40.

22. Wilson GJ, Murphy AJ, Pryor JF. Musculotendinous stiffness: its relationship to eccentric, isometric, and concentric performance. J Appl Physiol (1985). 1994;76(6):2714-9.

23. Bradley PS, Olsen PD, Portas MD. The effect of static, ballistic, and proprioceptive neuromuscular facilitation stretching on vertical jump performance. J Strength Cond Res. 2007;21(1):223-6.

24. Fowles JR, Sale DG, MacDougall JD. Reduced strength after passive stretch of the human plantarflexors. J Appl Physiol (1985). 2000;89(3):1179-88.

25. Martins A, Paz G, Vigário P, Silva GC, Maia M, Miranda H. Static stretching volume is associated with maximal repetition performance. J Exerc Physiol. 2014;17(6):24-33.

26. Brennecke A, Guimarães TM, Leone R, Cadarci M, Mochizuki L, Simão R, et al. Neuromuscular activity during bench press exercise performed with and without the preexhaustion method. J Strength Cond Res. 2009;23(7):1933-40.

27. Gentil P, Oliveira E, de Araújo Rocha Júnior V, do Carmo J, Bottaro M. Effects of exercise order on upper-body muscle activation and exercise performance. J Strength Cond Res. 2007;21(4):1082-6.

28. Connolly DA, Sayers SP, McHugh MP. Treatment and prevention of delayed onset muscle soreness. J Strength Cond Res. 2003;17(1):197-208.

29. Cheung K, Hume P, Maxwell L. Delayed onset muscle soreness : treatment strategies and performance factors. Sports Med. 2003;33(2):145-64.

30. McHugh MP. Recent advances in the understanding of the repeated bout effect: the protective effect against muscle damage from a single bout of eccentric exercise. Scand J Med Sci Sports. 2003;13(2):88-97 